\title{
Effects of energy drink major bioactive compounds on the performance of young adults in fitness and cognitive tests: a randomized controlled trial
}

\author{
Maximiliano Kammerer ${ }^{1 *}$, Jaime A Jaramillo ${ }^{2}$, Adriana García ${ }^{3}$, Juan C Calderón ${ }^{4}$ and Luis H Valbuena ${ }^{5}$
}

\begin{abstract}
Background: The consumption of beverages containing caffeine and taurine before exercising has been associated with increased physical and psychological performances and has been promoted to support the emotional state and provide vitality to consumers. However, there are contradictory results on these issues, it is not clear the effect of every major compound in relation to the whole effect of the beverages and there is a lack in knowledge about their degree of safety for consumption.

Methods: This study used a double-blind, placebo controlled, randomized, crossover design. Fourteen male volunteer soldiers from the Colombian army performed different tests to measure their cardiorespiratory fitness $\left(\mathrm{VO}_{2} \mathrm{max}\right.$ and maximum heart rate), time to exhaustion, strength (isometric strength), power (vertical jump), concentration (Grid test) and memory (Digits test) after drinking $250 \mathrm{ml}$ of one of the following beverages: one with $80 \mathrm{mg}$ caffeine, one with $1000 \mathrm{mg}$ taurine, one with $80 \mathrm{mg}$ caffeine plus $1000 \mathrm{mg}$ taurine, a commercial energy drink (Red Bull${ }^{\circledast}$ ) or a placebo drink. Subjects were caffeine-consumers that avoided caffeine during the day of evaluation. All beverages were matched in flavor and other organoleptic properties to the commercial one, were bottled in dark plastic bottles and were administered in identical conditions to the participants. Differences between treatments were assessed using repeated measures and analysis of variance.
\end{abstract}

Results: The mean \pm SD values of $\mathrm{VO}_{2} \mathrm{max}$, maximum heart rate, time to exhaustion, right handgrip strength, left handgrip strength, vertical jump, Grid test and Digits test were $61.3 \pm 6.2 \mathrm{ml} / \mathrm{kg} \cdot \mathrm{min}, 196 \pm 6.8$ beats per min, $17 \pm 1.2 \mathrm{~min}$, $56.8 \pm 6.6 \mathrm{kgf}, 53.1 \pm 5.9 \mathrm{kgf}, 41.1 \pm 3.8 \mathrm{~cm}, 19.9 \pm 5.9$ observed digits and $10.9 \pm 3.1$ remembered digits after drinking a placebo drink. Comparisons among the commercial drink, caffeine, taurine, caffeine plus taurine and placebo treatments did not show statistically differences in the results of the performed tests. No adverse effects were reported by the participants.

Conclusion: The consumption of caffeine $(80 \mathrm{mg})$ and taurine $(1000 \mathrm{mg})$ or their combination does not increase the physical and cognitive ability in young adults during exercise.

Keywords: Energy drink, Caffeine, Taurine, Physical condition, Cognitive ability

\section{Background}

Energy drinks are soft drinks, generally carbonated, composed of different ingredients such as caffeine, taurine, glucuronolactone, carbohydrates and vitamins with different rates of absorption to which the proposed effects of these beverages are attributed [1-3]. Furthermore, depending on

\footnotetext{
* Correspondence: nutricionendeporte@gmail.com

'Indeportes Antioquia and School of Nutrition and Dietetics, University of

Antioquia, UdeA, Calle 70 No 52-21, Medellín, Colombia

Full list of author information is available at the end of the article
}

the brand, these drinks have other ingredients such as amino acids, minerals and vegetable extracts, together with acidulants, preservatives, flavorings and colorings. These types of products have gained popularity worldwide since the 1990s, increasing exponentially the consumption among adolescents, athletes, and even senior citizens [4]. Manufacturers of these products promote their consumption with statements offering a variety of benefits among which increased physical performance, improved reaction rate, increased attention, higher concentration, 
improved emotional state and weight loss are included [4]. These are desirable characteristics for anyone, especially for active individuals. The interaction of the major bioactive compounds has been proposed as responsible for the alleged effects of these products; however, such statements have not been well studied and are not fully supported. Furthermore, there are contradictory results on the issue. Some studies attribute the alleged effects in improving the state of concentration and physical endurance to the combination of the compounds that make up these products $[5,6]$. Other studies point out that is very likely that most of the observed effects after consumption of these drinks are mainly produced by caffeine [7-9]. On the contrary, other studies have found no increase in the performance after consumption of these drinks [10].

Currently, many people seek benefits for their physical and mental health situation that has led them to use these types of drinks; nonetheless, despite an increased consumption by the general population, there is a lack of knowledge about the physiological effects of the compounds used in the formulations, the level of security in their consumption and their position within food standards. This is probably because there are few well-designed studies that provide accurate and conclusive findings on the subject.

Therefore, in this study we evaluated the efficacy and safety of energy drink consumption in a randomized, controlled, double-blinded, crossover trial and thus we were able to assess the direct involvement of caffeine and taurine, as major bioactive compounds of energy drinks, on physical and cognitive condition in young adults.

\section{Methods}

\section{Subjects}

A sample of 9 or 13 subjects was calculated in order to find differences in cognitive or physical tests. This was done by using PRIMER software 3.02 (PRIMER-E Ltd, United Kingdom), after establishing a confidence level of $95 \%$ and a power of $80 \%$. Nonetheless, 14 volunteered male soldiers, members of the National Army of Colombia Seventh Division, were included in the study. No cigarette smokers or users of psychoactive substances, or subjects under any medication (e.g. beta blockers) were included. People who consumed more than $600 \mathrm{ml}$ of coffee or more than 5 units of colas per day were excluded [11].

All participants underwent a complete physical examination and an electrocardiogram to determine their physical and mental conditions and all were diagnosed as healthy. In addition, they all signed the informed consent before the physical and cognitive tests. The procedures performed were endorsed and approved by the Indeportes Antioquia Research Committee and the National Army Research Committee in order to provide protection to the study participants.

\section{Experimental design}

This research used a double-blind, placebo controlled, randomized, crossover design, in which each participant visited six times the Exercise Physiology Laboratory from Indeportes Antioquia, in Medellín, Colombia, at $1540 \mathrm{~m}$ above sea level. Three fitness tests and two cognitive condition tests were applied in each visit. All evaluation sessions were separated between 48 or 72 hours and three subjects were evaluated per day. In the first session the subjects performed the tests, without drink consumption, to become familiar with their development. Testing was always conducted at the same time ( 2 pm-5 pm) in an environment with controlled temperature and humidity of $25^{\circ} \mathrm{C}$ and $60 \%$, respectively. A training specialist and a psychologist with extensive experience in sports fields were responsible for measuring all variables.

Participants maintained their regular food intake, workplaces and recreational activities during study participation, but did not train at high intensity eight hours before the tests, did not eat heavy meals two hours before the tests and avoided caffeine consumption during the day of evaluation. Since the participants were soldiers, they had a very controlled schedule regarding food and exercise every day. So we are sure that during the day of evaluation the variation in these variables was kept to the minimum. All subjects used appropriate clothing for physical exercise.

Before developing the tests all subjects were randomly assigned (with the help of Excel 2007, Microsoft Co, USA) to a treatment (a placebo drink, a caffeinated drink, a caffeine and taurine combined drink, a drink with only taurine, or a commercial energy drink) and after 45 minutes performed each of the tests. All subjects received all beverages during the study. The composition of the five beverages is illustrated in Table 1 .

The first four beverages were developed with a flavor profile similar to the commercial energy drink brand (Red Bull $\left.^{\oplus}\right)$. For the design and formulation of each treatment, final contents of other ingredients such as sweeteners, acidulants, stabilizers, and colorings were determined apart from the major bioactive components, to ensure a close level to the physicochemical properties such as acidity, $\mathrm{pH}$,

Table 1 Beverages composition per serving $(250 \mathrm{ml})$ used in the study

\begin{tabular}{llll}
\hline Drink number & Drink & Caffeine $(\mathbf{m g})$ & Taurine $(\mathbf{m g})$ \\
\hline 1 & Placebo & 0 & 0 \\
2 & Caffeinated drink & 80 & 0 \\
3 & $\begin{array}{l}\text { Drink with caffeine and } \\
\text { taurine }\end{array}$ & 80 & 1000 \\
4 & Drink with taurine & 0 & 1000 \\
5 & $\begin{array}{l}\text { Commercial drink } \\
\text { (Red Bull }{ }^{\oplus}\end{array}$ & 80 & 1000 \\
\hline
\end{tabular}


refractometric degrees, color, smell and sensory profile similar to the commercial energy drink.

Carbonated-based water was used for all drinks and the appropriate powder mixture, previously formulated, was added. All bioactive and additional ingredients were selected from certificated suppliers and were of food grade.

Brix (Mettler Toledo RE50 refractometer), $\mathrm{pH}$ and acidity (Mettler Toledo Seven Easy pH meter), and color (Hunter Lab D25LT colorimeter) were analyzed in all beverages.

In order to ensure similarity between all beverages, and keep the double-blind design of the study, organoleptic properties such as smell, color, sweetness, acidity, body and overall impression were evaluated. This analysis was supported by the Colcafé expert panel (Medellín, Colombia). Decisions on formulation adjustments were based on the results obtained and analyzed with FIZZ Sensory Analysis Software (France).

All treatment beverages, including the commercial one, were pasteurized at $80^{\circ} \mathrm{C}$ for 15 seconds, bottled in $250 \mathrm{ml}$ polyethylene terephthalate bottles, identified with numeric codes and administered in identical conditions to the participants. The day of the experiment, each participant consumed all $250 \mathrm{ml}$ of the assigned drink in a period of 10 to 15 minutes.

\section{Evaluations}

\section{Food consumption}

A 24-hour recall and an analysis of the complete menu provided by the Army to the soldiers were used to determine the dietary intake of the subjects in the study. Portion sizes, brands and cooking methods were recorded. We used the official food composition table from University of Antioquia's School of Nutrition and Dietetics (Medellín, Colombia) to determine the calories and nutrients consumed by the tested subjects and the average values were used in the analysis of consumption.

\section{Level of physical activity}

The level of physical activity was measured using the Global Physical Activity Questionnaire (GPAQ) of the World Health Organization [12]. Subjects were interviewed to remember the number of days from the last week when they did vigorous intensity or moderate intensity activities in three aspects (work or home activities, travel to different places or leisure activities) and the number of hours and minutes per day of different activities performed. This multidomain questionnaire was used since it was validated in both developed and developing countries and several populations including Latino people [13-15]. It is now used worldwide and this fact enables comparisons across diverse populations.

\section{Physical tests}

\section{Determination of cardiorespiratory fitness}

All participants completed a set of ergospirometry tests that allowed us directly monitoring their cardiorespiratory behavior. An open circuit spirometry (Oxycon Delta, Jaeger, Spain) previously calibrated was used. A ramp protocol was used in test performing [16]. After explaining the test to each subject, the resting ergospirometry parameters were taken and the test was started with a three minutes warming up period at a speed of 3.5 mile/h with a band inclination of $1 \%$, then the speed was increased by $0.5 \mathrm{mile} / \mathrm{h}$ every minute until exhaustion. To measure participant recovery, subjects continued walking with an intensity and duration similar to the warming up period for three minutes, followed by other two minutes of passive recovery in standing position. After the test each subject was disconnected from the computer and the information obtained was recorded. Maximum oxygen consumption $\left(\mathrm{VO}_{2} \mathrm{max}\right)$, maximum heart rate ( $\left.\mathrm{HRmax}\right)$ and time to exhaustion (TTE) were determined from test results.

\section{Strength calculation}

A static dynamometry test of the upper limbs was performed. We used handgrip strength, which has been considered a simple, fast and reliable test for measuring human strength in different populations [17-20]. For handgrip strength measurements (finger flexor force), a previously calibrated Takei Kiki Kogyo digital dynamometer (Japan), with a transducer 1270A, series 86002, $90 \mathrm{~kg}$, was used. Each of the participants performed a ten minutes guided warming up, focusing on the muscle groups that were evaluated. Subsequently, the evaluated subject stood up and held the transducer, in such a way that the upper end of this rests on the palm, under thenar and hypothenar regions, and the lower end on the second to the fifth finger second phalanx. Then, with the upper limb away from the body and no support points for the transducer, the soldier proceeded to perform bilateral maximum handgrip strength for five seconds; this procedure was repeated after one minute of recovery and the highest value was recorded [21].

\section{Power calculation}

Three vertical jumps were performed on a contact, previously calibrated, platform (Newtest Power timer series 10327) that calculated the flight time. Data obtained allowed to calculate the height reached and the subject mechanical power. Functioning of the test was explained to participants; then, there was a warming up emphasizing the muscles of the lower limbs. After subjects performed three nonconsecutive free jumps using arms, the height was measured by taking into account the flight 
time. Out of the three jumps performed the highest flight time was recorded [22].

\section{Cognitive tests}

\section{Focused attention test (Grid)}

This instrument allowed the evaluation of outside attention, both broad and narrow according to basic principles of attention control [23]. Its execution consisted of pointing out (according to the instructions provided previously) the numbers 1 to 38 that appeared in a grid with different sized digits, sequentially.

\section{Digit span WAIS subtest (Digits)}

Attention, resistance to distraction and immediate auditory memory were measured with a memory function subtest part of Wechsler Adult Intelligence Scale (WAIS) test. It consists of asking subjects to repeat two sets of numbers, which were previously read in direct and reverse order, respectively. A level of nine numbers in direct order and eight numbers in reverse order was used for the test. For this study we used the digit span test by changing the numbers in each sample to avoid learning, but keeping the series, times and original guidelines [24].

These are simple, of short-duration tests, which evaluate cognitive capacities important during the practice of different sports. Both tests have been previously used in similarly designed approaches to the research question of the present work (see [1]).

\section{Statistical analysis}

We checked the assumptions of normality, homogeneity and randomness of the different variables $\left(\mathrm{VO}_{2} \mathrm{max}\right.$, HRmax, TTE, right and left handgrip strength -RHS and LHS-, vertical jump, concentration and memory). The differences between treatments were measured using a $5 \times 5$ table (treatments $v s$. sessions) of repeated measures analysis of variance (ANOVA). The lower significant difference was developed by post-hoc analysis with the Tukey test. All data is reported in the text and the tables as mean \pm SD. Minitab 16.0 software was used to perform the analysis. A value of $\mathrm{p}<0.05$ was considered statistically significant.

\section{Results}

The 14 participants had an age of $20 \pm 1$ years (mean \pm SD), a weight of $68 \pm 7.2 \mathrm{Kg}$ and a height of $170.7 \pm$ $5.4 \mathrm{~cm}$. The total energy consumed by the soldiers was $2654 \pm 97 \mathrm{Kcal} /$ day and the percentage of it provided by carbohydrates, proteins and fat was 59\%, $14 \%$ and $27 \%$, respectively. Total hours spent in physical activity were also calculated and multiplied by the metabolic equivalent (MET) minutes per week to determine an energy expenditure of $4536 \pm 123 \mathrm{MET} / \mathrm{min} /$ week. Table 2 shows
Table 2 Level of physical activity of the soldiers involved in the study

\begin{tabular}{ll}
\hline Variable & Value \\
\hline Domestic work activities (min/day) & $60(15-129)^{*}$ \\
Movement to different places (min/day) & $52.5(30-60)^{*}$ \\
Movement activities & 12 participants \\
No movement activities & 2 participants \\
Recreational activities (min/day) & $25.7(15-40)^{*}$ \\
Sedentary behavior (min/day) & $60(60-90)^{*}$ \\
\hline
\end{tabular}

"Since these data were not normally distributed (Shapiro-Wilk test), medians and (interquartile ranges) are shown.

further characterization of the physical activity of the population.

The Table 3 presents the descriptive statistics of the physiological variables measured in the soldiers during the study. No significant differences were found in the values of $\mathrm{VO}_{2} \max (\mathrm{p}=0.736)$, in $\operatorname{HRmax}(\mathrm{p}=0.715)$, TTE $(\mathrm{p}=0.836)$, strength (RHS $\mathrm{p}=0.096$ and LHS $\mathrm{p}=$ $0.607)$, power $(\mathrm{VJ})(\mathrm{p}=0.066)$, concentration tests (Grid, $\mathrm{p}=0.763)$ and immediate memory (Digits, $\mathrm{p}=0.168$ ).

No adverse effects were reported by the subjects after drinking the beverages and during the time they were at the laboratory.

Our results suggest that there are not statistically significant differences among the results of the commercial drink, drinks with different bioactive compounds and placebo in the various tests carried out by the soldiers, at the delivered dose with a confidence level of $95 \%$.

\section{Discussion}

Our results indicate that the consumption of caffeine $(80 \mathrm{mg})$ and taurine $(1000 \mathrm{mg})$ in an energy drink before performing cognitive and fitness test has no effects on cardiorespiratory fitness indices $\left(\mathrm{VO}_{2} \max , \mathrm{HRmax}, \mathrm{TTE}\right)$, strength (RHS and LHS), power (VJ), concentration (Grid) and immediate memory (Digits).

We want to point out that all beverages were prepared and evaluated, previously to be administered to the soldiers, by an expert group of people with the help of industrial up-to-date technology and chemicals. So, their lack of effect on the studied variables cannot be proposed to result from inadequate quality of the beverages. Also, we want to make clear that the aim of the study was not to evaluate the effect of increasing doses of caffeine or taurine on the physical and cognitive performance, rather, it was to evaluate the effects of the amounts of these compounds currently found in most energy drinks available for free consumption.

Some of our findings contrast with those of others $[1,6,25,26]$. The effects have been attributed to the combination of ingredients like caffeine, taurine, glucuronolactone and $\mathrm{B}$ vitamins in these drinks. However, we 
Table 3 Descriptive statistics for the variables evaluated and the five treatments in all participants $(n=14)^{*}$

\begin{tabular}{lllllll}
\hline & Placebo & Caffeine & C + T & Taurine & Commercial drink & P value \\
\hline VO $_{2}$ max (ml/kg/min) & $61.3 \pm 6.2$ & $61.1 \pm 5.2$ & $61.8 \pm 5.4$ & $60.6 \pm 5.1$ & $61.1 \pm 5.1$ & 0.736 \\
HRmax (beats per $\mathbf{m i n})$ & $196 \pm 6.8$ & $196.5 \pm 4.5$ & $197.1 \pm 6$ & $197.8 \pm 6.6$ & $196.4 \pm 6.2$ & 0.715 \\
TTE (min) & $17 \pm 1.2$ & $17 \pm 1.03$ & $16.8 \pm 1.2$ & $16.9 \pm 1.4$ & $16.8 \pm 1.2$ & 0.836 \\
RHS (kgf) & $56.8 \pm 6.6$ & $55.3 \pm 6.7$ & $57.5 \pm 6.2$ & $55.4 \pm 6.9$ & $56.2 \pm 5.8$ & 0.096 \\
LHS (kgf) & $53.1 \pm 5.9$ & $53.5 \pm 4.9$ & $54 \pm 6.3$ & $52.6 \pm 7$ & $52.9 \pm 5.5$ & 0.607 \\
VJ (cm) & $41.1 \pm 3.8$ & $41.7 \pm 3.8$ & $41.5 \pm 4.2$ & $42.5 \pm 4.4$ & $40.9 \pm 4.1$ & 0.066 \\
GRID (number of observed digits) & $19.9 \pm 5.9$ & $19.8 \pm 4.4$ & $20.6 \pm 4.5$ & $19.1 \pm 4.7$ & $19.3 \pm 3.5$ & 0.763 \\
DIGITS (number of remembered digits) & $10.9 \pm 3.1$ & $10.6 \pm 2.9$ & $11.0 \pm 2.8$ & $11.2 \pm 2.7$ & $10.3 \pm 3.1$ & 0.168 \\
\hline
\end{tabular}

*Values are mean $\pm S D$. C $+T$ : caffeine + taurine, $V_{2}$ max: maximal oxygen uptake, HR: heart rate, TTE: time to exhaustion, RHS: right handgrip strength, LRH: left handgrip strength, $\mathrm{VJ}$ : vertical jump, GRID: concentration test, DIGITS: immediate memory test.

must clarify that in those studies the main ingredients of an energy drink (caffeine and taurine) were not isolated and the drinks were separated as control (placebo) and experimental (other drinks) groups without, in several cases, good masking of the treatments. In our study each subject was his own control which can minimize the bias introduced when evaluating compounds such as caffeine and taurine, since the individual response to the consumption of these substances may be different. Also, our methodology allowed a good masking of the treatments and gives strength to this study. On the other hand, our findings are consistent with the results obtained by Candow and colleagues [10], Umana-Alvarado and Moncada-Jiménez [27], and Nelson and colleagues [28] in which the consumption of the commercial beverage had not positively influenced the outcome of the physical tests performed. It is important to note that in our study we assessed the cardiorespiratory fitness directly (through ergospirometry) which can provide more reliable information than when tests are performed indirectly through a stress test.

Results shown in Table 3 demonstrate that the consumption of the commercial energy drink did not affect the HRmax of the evaluated subjects, in agreement also with the findings of Baum and Weiss [29], Bichler and coworkers [30] and the recent work of Nelson and colleagues [28]. In contrast, in Geiß and colleagues' work [6], lower HR values were reported after endurance athletes consumed a commercial energy drink. However, the subjects in the last research were athletes, while our study was conducted in a different population (soldiers). Since the soldiers studied have very similar dietary patterns, physical activity, employment and lifestyle (hours of sleep, free time management, level of education), the differentiating effect that could have these patterns on fitness and cognitive tests can be minimized.

In a recent report with similar design to ours, Nelson and colleagues [28] did not find any change in ride timeto-exhaustion and some cardiovascular performance variables on a sample of similar size of young, moderately fit subjects, after they drunk a caffeinated and taurinated commercial drink. Since we used subjects with higher $\mathrm{VO}_{2} \max$ values and the results were the same, it is tempting to speculate that the lack of effects of the studied compounds may persist in people within a relatively large range of physical capabilities.

On the other hand, a paper showed a significant increase in $\mathrm{VO}_{2} \max$, TTE and maximal workload in a stress test after consuming a taurine supplement [31]. The authors postulated that this could be due to attenuation of exercise-induced damage to deoxyribonucleic acid and increased exercise capacity by its protective properties at cellular level, which differs with the results found in this research. Unfortunately, we did not further explore in our subjects the mechanisms mentioned by that study [31].

In this research it was not found that the bioactive compounds have a positive effect on immediate memory and concentration tests, as opposite to several reports $[1,5,32,33]$, where the effect was attributed to either the caffeine or an ingredient combination. In those studies, however, each energy drink main compound was not evaluated. In our case, no differences were found in cognitive tests when combined or isolated bioactive compounds were consumed; possibly because either the amount of caffeine used is insufficient to positively influence the concentration and immediate memory test outcomes or there is not an actual effect. These results are consistent with findings made by others [34,35], who found no effects in the subject memory and alertness and concentration state. The dose we administered to the subjects was between the range of $1-2 \mathrm{mg} / \mathrm{kg}$ proposed by some authors to have physiological effects on several cognitive and physical functions when administered in energy drinks [3,36-38]. Although some authors have proposed that low doses of caffeine are associated with increased energy, alertness and vigilance, motivation and concentration, but not with memory in nonathletes [37], i.e., there is a task specific profile of response to caffeine, the variability in results obtained in 
active people with energy drinks suggests that this is not clear yet (see for instance [1,5,32 and results from the present work]). Further investigations are necessary for people who exercise to identify the lower caffeine doses that produce beneficial effects in their performance that can really be worthwhile [39-41], to evaluate the effect on several variables of cognitive performance more sensitive to low doses of caffeine [37] and also to study the potential additive effect of the components of energy drinks. The history of caffeine consumption and withdrawal in the subjects studied is not expected to affect our results, since in both low (about $50 \mathrm{mg} /$ day) and high caffeine (about $200 \mathrm{mg} /$ day) consumers, a period of withdrawal comparable to that used in the present work has no effect on the cognitive responses measured after a caffeine challenge $[37,42]$. The lack of influence of the consumer type on different variables affected or not by caffeine, has also been reported in works which involved people with a history of caffeine consumption ranging from zero to more than $200 \mathrm{mg} /$ day [26]. So, it is likely that caffeine habituation (from zero to $200 \mathrm{mg} /$ day) is not an important variable to explain results from studies evaluating the effect of energy drinks on sports performance.

It has also been suggested that taurine can help in skeletal muscle contractile function, by increasing the sarcoplasmic reticulum $\mathrm{Ca}^{2+}$ content and muscle force generation [43-46]. Moreover, the potential synergistic or additive effects of caffeine and taurine when administered in energy drinks deserve attention [3]. In this study, however, neither the drink with taurine, nor the taurine combined with caffeine nor the commercial drink favored a higher generation of strength than the placebo drink.

\section{Conclusions}

The different bioactive compounds studied here at the concentrations (caffeine $80 \mathrm{mg}$ and taurine $1000 \mathrm{mg}$ per serving of $250 \mathrm{ml}$ ) found in most currently available energy drinks do not have positive effects on cardiorespiratory fitness test $\left(\mathrm{VO}_{2} \mathrm{max}, \mathrm{HRmax}\right.$ and TTE), force or power; neither on concentration test nor on immediate memory in young, healthy, active adults. Results from the combination of beverage ingredients also showed no difference from the results obtained when consuming a placebo drink. No adverse effects were recorded after ingestion of the studied beverages.

\footnotetext{
Abbreviations

Digits: digit span WAIS subtest; Grid: focused attention test; GPAQ: Global Physical Activity Questionnaire; HRmax: maximum heart rate; LHS: left handgrip strength; MET: metabolic equivalent; RHS: right handgrip strength; TTE: time to exhaustion; $\mathrm{VJ}$ : vertical jump; $\mathrm{VO}_{2}$ max: maximum oxygen consumption; WAIS: Wechsler Adult Intelligence Scale.
}

\section{Competing interests}

The authors declare that they have no competing interests.

\section{Authors' contributions}

MK contributed to the conception and design, MK, JAJ, AG and LHV obtained the data, analyzed and interpreted it, JCC analyzed and interpreted the data. All authors drafted the manuscript and approved the final version.

\section{Acknowledgements}

The authors would like to thank the institutions involved in the study: Indeportes Antioquia, Colcafé, National Army of Colombia (Cuarta Brigada) and School of Nutrition and Dietetics from University of Antioquia, in Medellín, Colombia. We also want to thank Dr. Jaime Gallo, from University of Antioquia, for suggestions to the text.

\section{Author details}

${ }^{1}$ Indeportes Antioquia and School of Nutrition and Dietetics, University of Antioquia, UdeA, Calle 70 No 52-21, Medellín, Colombia. ${ }^{2}$ Colcafé S.A.S, Medellín, Colombia. ${ }^{3}$ Indeportes Antioquia, Medellín, Colombia. ${ }^{4}$ Physiology and Biochemistry Research Group-Physis, Department of Physiology and Biochemistry, Faculty of Medicine, University of Antioquia, Medellín, Colombia. ${ }^{5}$ Exercise Physiology Laboratory from Indeportes Antioquia and Physiology and Biochemistry Research Group-Physis, University of Antioquia, Medellín, Colombia.

Received: 9 April 2014 Accepted: 19 August 2014

Published online: 07 November 2014

\section{References}

1. Alford $\mathrm{C}$, Cox $\mathrm{H}$, Wescott $\mathrm{R}$ : The effects of red bull energy drink on human performance and mood. Amino Acids 2001, 21(2):139-150,

2. Seidl R, Peyrl A, Nicham R, Hauser E: A taurine and caffeine-containing drink stimulates cognitive performance and well-being. Amino Acids 2000, 19(3-4):635-642.

3. Campbell B, Wilborn C, La Bounty P, Taylor L, Nelson M, Greenwood M, Ziegenfuss T, Lopez H, Hoffman J, Stout J, Schmitz S, Collins R, Kalman D, Antonio J, Kreider R: International Society of Sports Nutrition position stand: energy drinks. J Int Soc Sports Nutr 2013, 10:1

4. Reissig C, Strain E, Griffiths R: Caffeinated energy drinks - a growing problem. Drug Alcohol Depend 2009, 99(1-3):1-10.

5. Scholey A, Kennedy D: Cognitive and physiological effects of an "energy drink": an evaluation of the whole drink and of glucose, caffeine and herbal flavouring fractions. Psychopharmacology 2004, 176(3-4):320-330.

6. Geiß K, Jester I, Falke W, Hamm M, Waag K: The effect of a taurine containing drink on performance in 10 endurance-athletes. Amino Acids 1994, 7(1):45-56.

7. Ballard S, Wellborn-Kim J, Clauson K: Effects of commercial energy drink consumption on athletic performance and body composition. Phys Sportsmed 2010, 38(1):107-117

8. Ivy J, Kammer L, Ding Z, Wang B, Bernard J, Liao Y, Hwang J: Improved cycling time-trial performance after ingestion of a caffeine energy drink. Int J Sport Nutr Exerc Metab 2009, 19(1):61-78.

9. Childs $\mathrm{E}$, de Wit H: Enhanced mood and psychomotor performance by a caffeine-containing energy capsule in fatigued individuals. Exp Clin Psychopharmacol 2008, 16(1):13-21.

10. Candow D, Kleisinger A, Grenier S, Dorsch K: Effect of sugar-free Red Bull energy drink on high-intensity run time-to-exhaustion in young adults. J Strength Cond Res 2009, 23(4):1271-1275.

11. Griffiths R, Juliano L, Chausmer A: Caffeine: Pharmacology and Clinical Effects. In Principles of Addiction Medicine. 3rd edition. Edited by Graham A Schultz T, Mayo-Smith M, Ries R, Wilford B. Chevy Chase, MD: American Society of Addiction Medicine; 2003:193-224.

12. World Health Organization: Global Physical Activity Questionnaire (GPAQ). Last visit October 2013. [http://www.who.int/chp/steps/GPAQ/en/]

13. Bull F, Maslin T, Armstrong T: Global physical activity questionnaire (GPAQ): nine country reliability and validity study. J Phys Act Health 2009, 6:790-804.

14. Trinh $\mathrm{O}$, Nguyen $\mathrm{N}$, van der Ploeg $\mathrm{H}$, Dibley $\mathrm{M}$, Bauman $\mathrm{A}$ : Test-retest repeatability and relative validity of the Global Physical Activity Questionnaire in a developing country context. J Phys Act Health 2009, 6(Suppl 1):S46-S53. 
15. Hoos T, Espinoza N, Marshall S, Arredondo E: Validity of the global physical activity questionnaire (GPAQ) in adult Latinas. J Phys Act Health 2012, 9(5):698-705

16. López-Chicharro J, Fernández-Vaquero A: Fisiología del ejercicio. 3rd edition. Madrid, España: Editorial Médica Panamericana; 2006.

17. Moreira D, Aiza R, de Godoy J, do Nascimento A: Abordagem sobre preensão palmar utilizando o dinamômetro JAMAR: uma revisão de literatura. Rev Bras Ci e Mov 2003, 11:95-99.

18. Godoy J, Barros J: Avaliação da força de preensão palmar e composição corporal em portadores da trissomia 21 no Distrito Federal. Lect Educ Fís Deportes 2005, 89. [http://www.efdeportes.com/efd89/palmar.htm]

19. Sasaki H, Kasagi F, Yamada M, Fujita S: Grip strength predicts cause-specific mortality in middle-aged and elderly persons. Am J Med 2007, 120(4):337-342.

20. Rantanen T, Volpato S, Ferrucci L, Heikkinen E, Fried L, Guralnik J: Handgrip strength and cause-specific and total mortality in older disabled women: exploring the mechanism. J Am Geriatr Soc 2003, 51(5):636-641.

21. Valbuena R: Aplicación de un índice de fuerza isométrica máxima (Morhause) a un grupo de deportistas de rendimiento. Rev Ant Med Dep 1999, 2:18-23.

22. Bosco C: La valoración de la fuerza con el test de Bosco. Barcelona: Paidotribo; 1994

23. Nideffer R, Sharpe R: ACT: Attention Control Training. New York: Wyden Books; 1978

24. Zimmerman I, Woo-Sam J, Glasser A: Interpretación Clínica de la Escala de Inteligencia de Wechsler para Adultos (WAIS). Madrid: TEA ediciones; 1981.

25. Rahnama N, Gaeini A, Kazemi F: The effectiveness of two energy drinks on selected indices of maximal cardiorespiratory fitness and blood lactate levels in male athletes. J Res Med Sci 2010, 15(3):127-132.

26. Forbes S, Candow D, Little J, Magnus C, Chilibeck P: Effect of Red Bull energy drink on repeated Wingate cycle performance and bench-press muscle endurance. Int J Sport Nutr Exerc Metab 2007, 17(5):433-444.

27. Umana-Alvarado M, Moncada-Jiménez J: The effect of an energy drink on aerobic performance in male athletes. Med Sci Sports Exer 2004, 36(5):S174-S175.

28. Nelson M, Biltz G, Dengel D: Cardiovascular and ride time-to-exhaustion effects of an energy drink. J Int Soc Sports Nutr 2014, 11(1):2.

29. Baum M, Weiss M: The influence of a taurine containing drink on cardiac parameters before and after exercise measured by echocardiography. Amino Acids 2001, 20(1):75-82.

30. Bichler A, Swenson A, Harris M: A combination of caffeine and taurine has no effect on short term memory but induces changes in heart rate and mean arterial blood pressure. Amino Acids 2006, 31(4):471-476.

31. Zhang M, Izumi I, Kagamimori S, Sokejima S, Yamagami T, Liu Z, Qi B: Role of taurine supplementation to prevent exercise-induced oxidative stress in healthy young men. Amino Acids 2004, 26(2):203-207.

32. van den Eynde F, van Baelen P, Portzky M, Audenaert $K$ : The effects of energy drinks on cognitive performance [Article in Dutch]. Tijdschr Psychiatr 2008, 50(5):273-281

33. Fredholm B, Bättig K, Holmén J, Nehlig A, Zvartau E: Actions of caffeine in the brain with special reference to factors that contribute to its widespread use. Pharmacol Rev 1999, 51:83-133.

34. Warburton D, Bersellini E, Sweeney E: An evaluation of a caffeinated taurine drink on mood, memory and information processing in healthy volunteers without caffeine abstinence. Psychopharmacology 2001, 158(3):322-328.

35. Lombardo J: Supplements and athletes. South Med J 2004, 97(9):877-879.

36. Cox G, Desbrow B, Montgomery P, Anderson M, Bruce C, Macrides T, Martin D, Moquin A, Roberts A, Hawley J, Burke L: Effect of different protocols of caffeine intake on metabolism and endurance performance. J Appl Physiol 2002, 93(3):990-999.

37. Hewlett $P$, Smith $A$ : Acute effects of caffeine in volunteers with different patterns of regular consumption. Hum Psychophamacol Clin Exp 2006, 21:167-180.

38. Goldstein E, Ziegenfuss T, Kalman D, Kreider R, Campbell B, Wilborn C, Taylor L, Willoughby D, Stout J, Graves B, Wildman R, Ivy J, Spano M, Smith A, Antonio J: International Society of Sports Nutrition Position Stand: caffeine and performance. J Int Soc Sports Nutr 2010, 7(1):5.

39. Burke L, Cort M, Cox G, Crawford R, Desbrow B, Farthing L, Minehan M, Shaw N, Warnes O: Supplements and sports foods. In Clinical Sports Nutrition. 3rd edition. Edited by Burke L, Deakin V. Sydney: McGraw-Hill; 2006.

40. Bell D, McLellan T: Effect of repeated caffeine ingestion on repeated exhaustive exercise endurance. Med Sci Sports Exerc 2003, 35:1348-1354.
41. Doherty $M$, Smith $P$ : Effects of caffeine ingestion on rating of perceived exertion during and after exercise: a meta-analysis. Scand J Med Sci Sports 2005, 15:69-78.

42. Hewlett $P$, Smith $A$ : Effects of repeated doses of caffeine on performance and alertness: new data and secondary analyses. Hum Psychophamacol Clin Exp 2007, 22:339-350.

43. Mueller E, Rado L, Weise M, Cass T: Effects of red bull on Wingate testing of college aged students. J Undergrad Kin Res 2007, 2(2):12-18.

44. Kim W: Debunking the Effects of Taurine in Red Bull Energy Drink Nutrition Bytes 2003, 9(1). Available at: http://www.escholarship.org/uc/item/ $65 \mathrm{k} 8 \mathrm{r} 3 \mathrm{bd}$.

45. Bakker A, Berg $\mathrm{H}$ : Effect of taurine on sarcoplasmic reticulum function and force in skinned fast-twitch skeletal muscle fibres of the rat. J Physiol 2002, 538:185-194.

46. Cuisinier C, Ward R, Francaux M, Sturbois $X$, de Witte $P$ : Changes in plasma and urinary taurine and amino acids in runner immediately and $24 \mathrm{~h}$ after a marathon. Amino Acids 2001, 20:13-23.

doi:10.1186/s12970-014-0044-9

Cite this article as: Kammerer et al:: Effects of energy drink major bioactive compounds on the performance of young adults in fitness and cognitive tests: a randomized controlled trial. Journal of the International Society of Sports Nutrition 2014 11:44.

\section{Submit your next manuscript to BioMed Central and take full advantage of:}

- Convenient online submission

- Thorough peer review

- No space constraints or color figure charges

- Immediate publication on acceptance

- Inclusion in PubMed, CAS, Scopus and Google Scholar

- Research which is freely available for redistribution

Submit your manuscript at www.biomedcentral.com/submit
C) Biomed Central 